$\xi=-1$

\title{
Detection and classification of wipe transitions in sport videos in presence of object motion
}

\author{
Dr. Salim Chavan ${ }^{1 *}$, Dr. M. Narayana ${ }^{2}$, Dr. L Koteswara Rao ${ }^{2}$ \\ ${ }^{1}$ Wainganga College of Engineering and Management, Nagpur, India \\ ${ }^{2}$ Department of ECE,Vardhaman College of Engineering,Hyderabad,India \\ *Corresponding author E-mail: salimsahil97@ rediffmail.com
}

\begin{abstract}
Transition detection is the necessary step in retrieval and investigation of videos on the basis of contents. Since last two decades, most of the researchers are involved in developing algorithms for detection of gradual transitions. However, the features of all gradual transitions are different and hence this issue still needs to be addressed precisely. After identifying this issue, an integrated shot boundary detection method is proposed in this paper. Wipe transition is the effect which is mostly used in the video making industries .Since there are different types of wipe transitions, it becomes very difficult to detect such transitions. Due to complexity in detection of wipes due to noise, object and camera motion earlier methods have less focus on this issue. In this paper an efficient wipe detection method is presented which gives better results even in the presence of object and camera movements.
\end{abstract}

Keywords: Wipe Transition Effect; DWT; Camera Motion; Object Motion; LT.

\section{Introduction}

Computers and video search engines should be well equipped to make it possible to find and access relevant information from this bulky data. Mostly all the video search engines are still based on textual search and are dependent on manual annotation of the data. Manual annotation is very time consuming, costly and sometimes inaccurate because the annotations always depend on the cultural background, language and opinions of the annotators. Thus Automatic annotation methods are required to access all the video data available.

There are multiple ways by which temporal segmentation can be performed. However, most of the time we are interested in much shorter time scales. If we want to do searches inside a digital video, we must calculate features for some smaller parts of the show since the search can be directed only towards those segments for which we have numerical data in a usable form. The length of the video segments defines the shortest time scale we can use in our searches. On the other hand, the length of the segments should neither be too short. For example most of the time it would not be possible to calculate and store a feature vector for each frame since there are prohibitively many frames in long videos. The visual low-level feature vectors typically do not change that much between consecutive frames within a single shot, a continuous video segment filmed in a single camera run. Thus, it is beneficial to handle these groups of similar frames as one block instead of as a list containing dozens of nearly identical frames. Shots can therefore be defined to be the shortest video segments that are practical to use in video retrieval systems. Moreover, it seems instinctively legitimate to use shots as the basic elements in the video retrieval task since they are the elementary building blocks used by video production houses while making movies. Using shots, or some similar slightly longer video segments, in the search task can also be justified intuitively by claiming that the users usually search for events in the video instead of single frames. It is obvious that such events typically take place at the shot level or in the scene level, which is hierarchically the next structural level in videos above the shots. To be able to segment video according to shot or scene boundaries, a "shot boundary detector" is needed. Automatic solving of shot boundaries is easier than solving of scene boundaries and that is why shots are usually used in content-based video retrieval. However, if we want to detect the semantically higher scene level boundaries, shot boundary information is still highly useful since scenes are made of shots. In video processing domain shot boundary detection is a field of research. In video analysis and proficient browsing, it requires to break the video sequences into appropriate segments called as shots. At the time of editing the video some effects are added between the two shots. Computer vision techniques have recently received increasing attention among researchers to detect the shot transition automatically. Shot transition may involve different effects such as fades, dissolves and wipes.

Fades: In fade-in brightness is slowly increasing till we get a bright frame and in a fade-out brightness is slowly decreasing till we get a black frame.

Dissolves: It is the result of superimposition of fade in and fade out.

Wipes: In wipe one frame is replaced by another with some special shape. In this, a wipe a line moves on the screen from one end to another end of screen. Wipes can be further divided into various kinds of sub types such as vertical wipe, horizontal wipe, diagonal wipe ,square wipe, star wipe, clock wipe and many more depending on how the line moves, . 

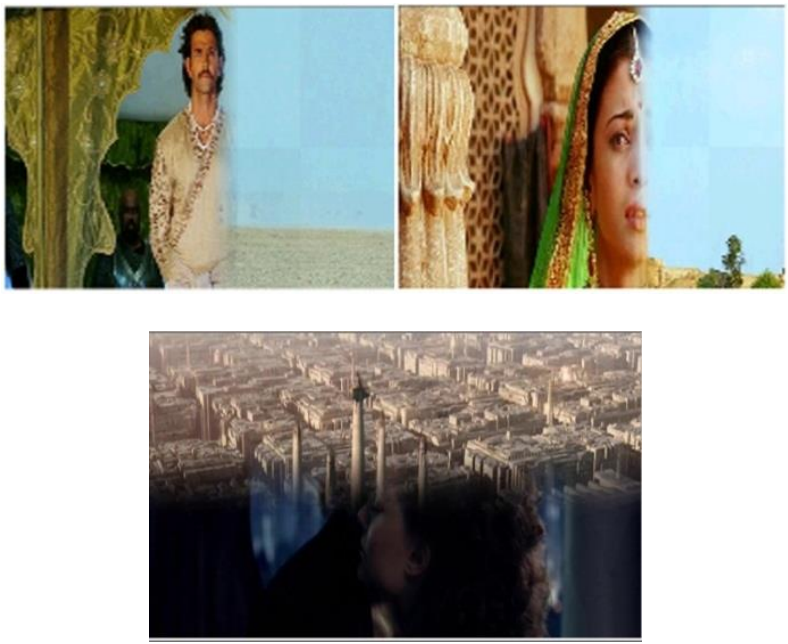

Fig. 1: Vertical and Horizontal Wipe Frames.

\section{Related work}

In [1] Zhe-Ming Lu et al proposed the method of detecting shot boundary using SVD and Pattern Matching. For fast detection, the design approach presented a selection of candidate segment as well as singular value decomposition. Using adaptive thresholds, the positions and length of gradual transitions are predicted leaving non-boundary frames. Colour histograms in the HSV is obtained for every frame in each candidate segment, forming a frame-feature matrix. Transition detection is then performed on the frame-feature matrices of all segment which minimise the feature aspects, giving a new technique for shot boundary detection. It achieves a better detection speed and accuracy. A Quick Algorithm for searching and detecting shot changes in video is proposed by Ehsan Amini, Somayyeh Jafarali Jassbi et al [2] . This method uses a divide and conquer algorithm which is able to carry out fast search for shot change probability. Algorithm divides the video into two parts and continues the search in each part. Worst condition, is when there are lot of shot changes in video. This algorithm is not capable of detecting the shot boundaries located inside a long scene. Sadik Fanan et al [3] used the mean of statistical image differences which allows dynamic threshold calculations to determine the wipe transition efficiently. It could detect only few types of wipes and in case of rapid object motion, the performance is not satisfactory. Shan Li et al[4] proposed an unique wipe detection algorithm that can detect most wipe effects with accurate frame ranges. Wipe based on its nature is carefully modelled to eliminate possible false positives due to motion. More accurately, to distinguish an ideal wipe, properties of independence and completeness are suggested. If every pixel changes its value only once it is the property of independence, whereas the property of completeness means that all pixels will have their values altered on completion of an ideal wipe transition. Adnan M. Alattar et al [5] proposed a model to derive the statistical characteristic of the frames in the wipe region. In this, it is stated by the author that the means and the variances of the frames in the wipe region possess either a linear or quadratic behaviour. By calculating the first derivative of the curve of the means and the variances both, the linear behaviour is detected and by calculating the second derivative of the curve of the means and curve of the variances the quadratic behaviour is detected. Min Wu et. al [6] have proposed algorithm, in which wipes are detected using both structural and statistical feature. An interesting method presented by T.-C. Pong et al. [8] for wipe detection is based on spatial and temporal slice analysis. There are matching patterns on the spatio-temporal slices for different styles of wipes. Based on these observations, the authors altered wipe detection to the specific patterns recognition on spatio-temporal slices. The proposed algorithm has shown good results when dealing with simple wipe types such as horizontal and vertical wipes however the perfor- mance of the algorithm falls exponentially when dealing with complex wipe type.

A wipe detection method is proposed by U. Naci et al. [9] in which two consecutive shots before and after wipe are spatially well separated at any time. This literature addresses the detection of horizontal and vertical wipes only. According to wipe detection method proposed by Zabih et al. [10], wipes reveal a typical pattern in the ECR time series. This pattern can be exploited to detect the potential wipe effects. The proposed algorithm has shown less immunity towards noise and the performance decrease when used over different genres of videos. According to a research published by R. Lienhart et al [7], for wipes, the adjacent shots are spatially well separated but not temporally separated. The spatial separation of shots is exploited by the proposed algorithm to effectively detect the potential wipe frames.

Alattar A.M et al [12] put forward a method of wipe detection based on the changes in the means and the variances of wipe region frames. This however suffers from high false positives causing due to motion. K.K Warhade et al [13] suggested a technique using pre-processing followed by detection and classification by Hough transform. Hang Bin et al [14] proposed a method for wipe detection using 3-D wavelet transforms and motion vector. This uses the Global motion compensation to minimise the effects of motions.

Fernando W.A.C. et al [15] proposed an approach which decomposes each frame into low and high-resolution components which after analysing and further recombining together forming a wipe transition detector. In this, mapping of each image with the reduced image is done. Then, using structural properties and statistical features of the images wipe transition region is recognised. Finally, Hough transform is used to analyze the wipe patterns and the direction of wipe. The results shows that this method is detecting all wipe regions precisely even though video sequence contains other special effects. Kwang Deok Seo et al [16] proposed wipe detection algorithm based on visual rhyme spectrum in which authors suggested that Visual Rhythm Spectrum contains distinctive visual features for various video effects. The proposed method is employed with the partial data of digital compressed video. This idea can be unanimously applied to various shotchange categories such as scene-cuts and wipes.

This method is put forward by Mark S. Drew et al [17]. In this, the authors used the available image pixels to generate images in spatio- temporal domain. This algorithm computes a 2-D histogram based on chromaticity, and then intersects that histogram with that of the previous frames one or several frames earlier. It results into an image containing cuts and wipes as very strong edges, almost once in a background of zeroes. This method does not take into account object and camera motion.

\section{Methodology}

Our algorithm for wipe transition detection is based on DWT. Each frame in the video is passed through DWT for feature extraction. This is done by passing each frame through many filter banks followed by a decimator. Filter banks consists of low and high pass filters at each decomposition stage,. This filter bank splits signal into two sub bands. The low pass filter meant for averaging operation which extracts the common information of the video signal. The high pass filter meant for differentiating operation which extracts the detailed information of the video signal. The output of the filtering operation is thus decimated by 2. A 4-level wavelet decomposition of a video frame is shown in below fig. 2 . 
(A)
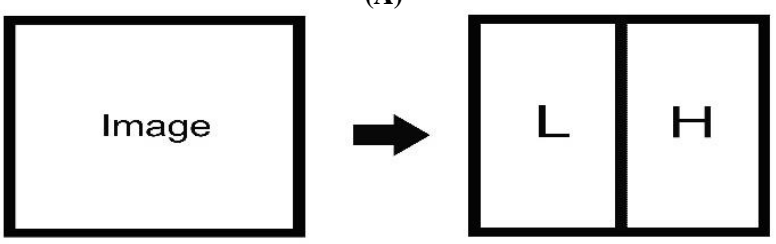

(B)
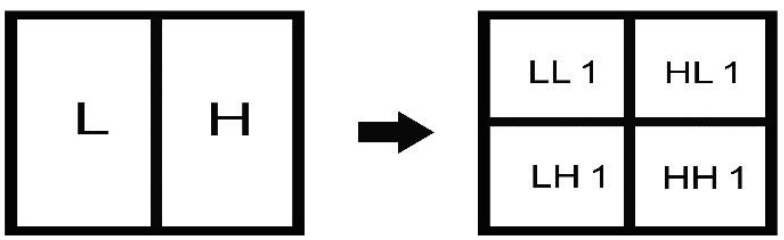

(C)

\begin{tabular}{|c|c|}
\hline LL1 & HL1 \\
\hline LH1 & HH1 \\
\hline
\end{tabular}

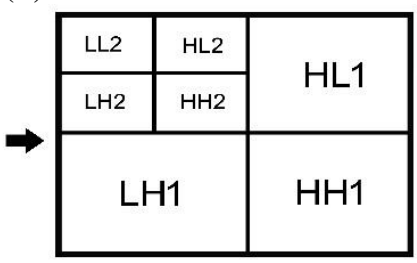

(D)

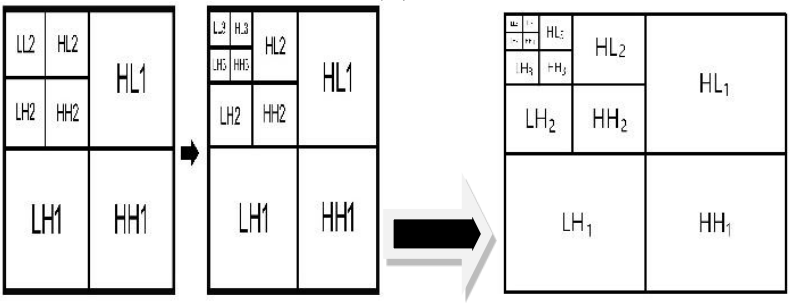

Fig. 2: (A) Horizontal Transform Two Sub Bands (B) Vertical Transforms Four Sub Bands (C) Second Level Filtering (D) Second, Third and Fourth Level Filtering.

\section{Algorithm steps}

This proposed Algorithm for detection of various wipes is explained as follows.

1) Each frame is changed from RGB to Gray level scale followed by decomposition to 4 level using DWT.

2) For each frame, the mean of approximate wavelet coefficient is calculated and is plotted with respect to No. of frames for video clip on test. Some sample graphs for different videos and different wipes are shown in fig. 3 below.

(a) Clock wise circular wipe present from frames 143 to173 $=30$

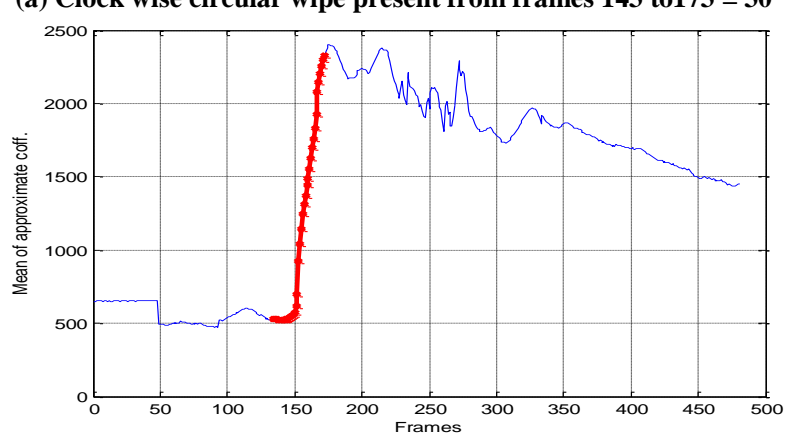

(b). Squared wipe present from frames 238 to $267=31$

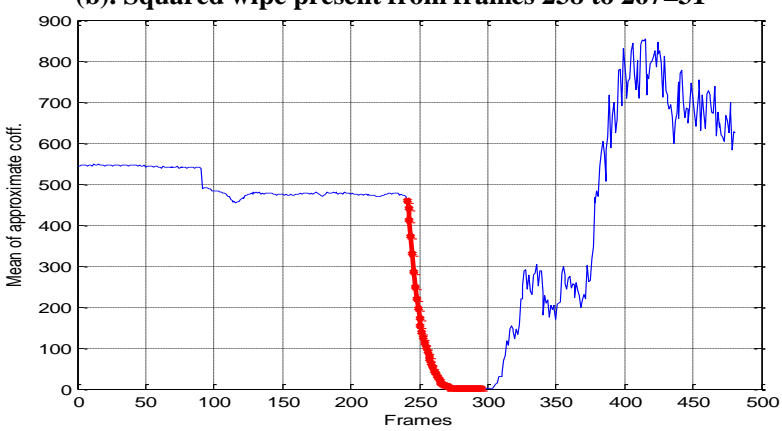

(C). Horizontal Wipe Present from Frames 156 To 187=31

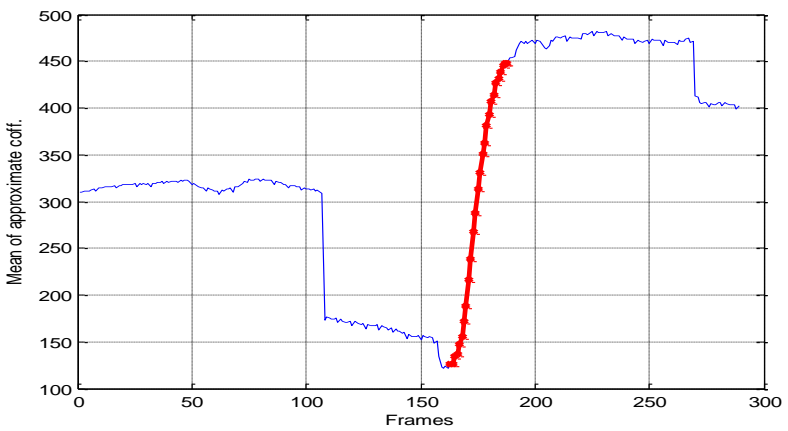

Fig. 3: (A), (B) and (C) Variation in Value of Mean of Approximate Wavelet Coefficient vs. Number of Frame.

3) It can be seen from the graph that there is a gradual increase or decrease of values if wipe is present for more than consecutive 20-30 frames.

4) We may get a short gradual increase or decrease in the graph for short duration of time in case of camera motion or object motion, as shown in the graph.Sometimes camera motion or object motion are also detected as wipe.

5) To differentiate between camera motion, object movements from wipe, we have to ensure if no. Of frames are more. If it is more means it belongs to motion.

6) Our algorithm examines all kinds of wipes transitions very effectively.

\section{Performance evaluation}

Almost all of the algorithm developer who has worked in shot boundary detection has used Recall and Precision as the measures for testing algorithm performance. Recall means how accurately the wipes are detected by algorithm. Precision gives the accuracy of the algorithm to minimize the false positives which occurs because of object and camera motion.

Performance metrics are mathematically defined as

Reall $=\frac{\mathrm{C}}{(\mathrm{C}+\mathrm{M})}$

Where $\mathrm{C}$ denotes correct detections and $\mathrm{M}$ denotes missed detections

Precision $=\frac{\mathrm{C}}{(\mathrm{C}+\mathrm{F})}$

Where $\mathrm{F}$ denotes false detections

In addition to Recall and Precision many of the studies have used two more performance criterias, those are F1 measure and Retrieval Success Index (RSI). RSI combines correct detections, false detection and miss detections to give in a single platform for performance evaluation base.

F1 measure and RSI are defined mathematically as

$\mathrm{F} 1$ measure $=2 * \frac{(\mathrm{R} * \mathrm{P})}{(\mathrm{R}+\mathrm{P})}$ 
And

Retrieval Success Index $=\frac{\mathrm{C}}{(\mathrm{C}+\mathrm{M}+\mathrm{F})}$

\section{Result and analysis}

The developed algorithm was tested and implemented on a number of selected sport videos. Performance and accuracy of algorithm can be validated by comparing the wipe transitions detected manually and by using the proposed Quick wipe algorithm. Table 1 below shows the Correct, False $\&$ Missed detections.

Table 1: Proposed Algorithm results

\begin{tabular}{lllll}
\hline Video Name & $\begin{array}{l}\text { Total } \\
\text { No .of } \\
\text { frame } \\
\text { under } \\
\text { test }\end{array}$ & $\begin{array}{l}\text { Correct } \\
\text { Detections }\end{array}$ & $\begin{array}{l}\text { Miss Detec- } \\
\text { tions }\end{array}$ & $\begin{array}{l}\text { False Detec- } \\
\text { tions }\end{array}$ \\
\hline $\begin{array}{l}\text { India-SA } \\
\begin{array}{l}\text { Match } \\
\text { India- }\end{array}\end{array}$ & 12500 & 705 & 38 & 24 \\
$\begin{array}{l}\text { Neuziland } \\
\text { Match }\end{array}$ & 10225 & 558 & 35 & 41 \\
$\begin{array}{l}\text { India- } \\
\text { Srilanka } \\
\text { Match }\end{array}$ & 5600 & 220 & 14 & 11 \\
\hline
\end{tabular}

From above results Recall, Precision and the F1 measure of the algorithm can be tabulated as below

Table 2: Recall, Precision and F1 measure

\begin{tabular}{llllll}
\hline Movie Name & Recall & Precision & F1 Measure & RSI \\
\hline India-SA Match & 94.88 & 96.70 & 95.78 & 91.91 \\
India-Neuziland Match & 94.09 & 93.15 & 93.61 & 88 \\
India- Srilanka Match & 94.01 & 95.23 & 94.61 & 89.79 \\
\hline
\end{tabular}

Table 3: Comparison with Previous Algorithms

\begin{tabular}{lllll}
\multicolumn{5}{c}{ Table 3: Comparison with Previous Algorithms } \\
\hline Algorithm & Video & Video 1 & Video 2 & Video 3 \\
\hline \multirow{3}{*}{ Proposed } & $\mathrm{R}$ & 94.88 & 94.09 & 94.01 \\
& $\mathrm{P}$ & 96.70 & 93.15 & 95.23 \\
& $\mathrm{~F} 1$ & 95.78 & 93.61 & 95.09 \\
Alattar[10] & $\mathrm{R}$ & 68.60 & 78.57 & 71.23 \\
& $\mathrm{P}$ & 72.31 & 77.03 & 78.03 \\
& $\mathrm{~F} 1$ & 65.92 & 78.20 & 79.49 \\
Fernando et al.[1] & $\mathrm{R}$ & 56.19 & 53.04 & 67.35 \\
& $\mathrm{P}$ & 86.72 & 83.52 & 86.30 \\
& $\mathrm{~F} 1$ & 61.77 & 64.36 & 72.74 \\
Nam et al.[2] & $\mathrm{R}$ & 62.65 & 78.53 & 86.78 \\
& $\mathrm{P}$ & 92.40 & 91.83 & 90.12 \\
& $\mathrm{~F} 1$ & 78.19 & 83.42 & 87.45 \\
\hline
\end{tabular}

The Recall and Precision obtained from the algorithm are given in the table 2 which evaluate the performance of an algorithm. On the basis of the above results it can be said that the algorithm gives high performance and accuracy of wipe transition detections. Our proposed methodology efficiently reduces the false positives occurred because of the presence of object and camera motions.

\section{Conclusions and future scope}

In this paper we proposed an efficient technique for detection and classification of different wipe effects in the videos related to sports. The detection of wipe transitions seem to be more complicated as compared to detection of hard cut transition and other gradual transitions which are simple and easy to detect. For this reason, we addressed the issue of wipe transition detection. In order to detect wipe transitions, we used db6 Discrete Wavelet Transform. Different number of video clips have been chosen for the experimentation purpose. It is clear from the results that the algorithm gives accurate detection of wipe transitions. However the algorithm performance degrades in presence of the camera and object motion which lasts for more than 30 consecutive frames which is very rare case. Overall, this algorithm can be used to enlarge targeted study aimed at shot boundary detection. In future, we can extend our work for detecting more wipe patterns even in the presence of rapid object motion, illumination and fire flickers.

\section{References}

[1] Zhe-Ming Lu,'Fast video shot Boundary Detection Based on SVD and Pattern matching", image processing ,IEEE on (Volume 22,issue : 12) September 2013

[2] Ehsan Amini, Somayyeh Jafarali Jassbi, "A Quick Algorithm to Search and Detect Video Shot Changes", "International Journal of Computer Applications, Volume 115 - No. 3, April 2015".

[3] Salim Chavan, Sadik Fanan, Dr. Sudhir Akojwar "An Efficient Method for Detection of Wipes in Presence of Object and Camera Motion", "International Journal of Innovative Research in Advanced Engineering (IJIRAE) ISSN: 2349-2163 Volume 1 Issue 8 , September 2014".

[4] Shan Li, Moon chuen Lee, "Effective Detection of Various Wipe Transitions"," IEEE transactions on circuits and systems for video technology, vol. 17, no. 6, June 2007" https://doi.org/10.1109/TCSVT.2007.896621.

[5] V.Kumthekar, Prof. J.K. Patil "Key frame extraction using color histogram method", International Journal of Scientific Research Engineering \& Technology (IJSRET) Volume 2 Issue 4 pp 207 214 , ISSN 2278 - 0882, july- 2013

[6] M. Wu, W. Wolf, and B. Liu, "An algorithm for wipe detection," in Proc. ICIP, 1998, pp. 893-897.

[7] Lienhart R., "Reliable transition detection in videos: a survey and practitioner's guide", Int. J. Image Graph, vol. 1, no. 3, pp. 469-486, 2001. https://doi.org/10.1142/S021946780100027X.

[8] C.W. Ngo, T.-C. Pong, and R. T. Chin, "Video partitioning by temporal slice coherency," IEEE Trans. Circuits Syst. Video Technol., vol. 11, no. 8, pp. 941-953, Aug. 2001. https://doi.org/10.1109/76.937435.

[9] U. Naci and A. Hanjalic, "TU Delft at TRECVID 2005: Shot boundary detection," in Proc. TRECVID 2005 Workshop, 2005

[10] R. Zabih, J. Miller, and K. Mai. A Feature-Based Algorithm for Detecting and Classifying Scene Breaks. Proc.ACM Multime- dia 95, San Francisco, CA, pp. 189-200, Nov. 1995 https://doi.org/10.1145/217279.215266.

[11] Robert A. Joyce, Bede Liu, "Temporal Segmentation of Video Using Frame and Histogram Space", "IEEE transactions on multimedia, vol. 8, no. 1, february 2006" https://doi.org/10.1109/TMM.2005.861285.

[12] Alattar A.M., "Wipe scene change detector for segmenting uncompressed video sequences"," Circuits and Systems, 1998. IS- CAS '98. Proceedings of the 1998 IEEE International Symposium on (Volume:4 )"

[13] K. Warhade, S.N. Merchant, U.B. Desai, "Effective algorithm for detecting various wipe patterns and discriminating wipe from object and camera motion",' Image Processing, IET (Volume:4 , Issue: 6 ) 2010"

[14] Bing Han, Hongbing Ji, Xinbo GAO, "A 3D wavelet and motion vector based method for wipe transition detection"," Signal Processing, 2004. Proceedings. ICSP '04. 2004 7th International Conference , 2004"

[15] W.A.C. Fernando, C.N. Canagarajah,D.R.Bull, "Wipe scene change detection in video sequences", "Image Processing, 1999. ICIP 99. Proceedings. 1999 International Conference" https://doi.org/10.1109/ICIP.1999.817120.

[16] Kwang-deok Seo, Seong Jun Park, and Soon-heung Jung, "Video Partitioning by Temporal Slice Coherency", "IEEE Transac- tions on Consumer Electronics, Vol. 55, No. 2, MAY 2009"

[17] Drew M. S., Li Z-N, and Zhang X., "Video dissolve and wipe detection via spatio-temporal images of chromatic histogram differences", In Proc. IEEE Int. Conf. Image Process, vol. 3, pp. 929-932, 2000. https://doi.org/10.1109/ICIP.2000.899609. 